\title{
Eficácia de Atrativos Alimentares na Captura de Zaprionus indianus (Gupta) em Pomar de Figo em Santa Maria - RS
}

\author{
Mauricio Paulo Batistella Pasini ${ }^{\bowtie}$, Dionísio Link \& Diniz Fronza²
}

1. Universidade Federal de Santa Maria, e-mail: mauricio.pasini@gmail.com (Autor para correspondência ${ }^{\bowtie}$ ), dionisiolink@yahoo.com.br. 2. Colégio Politécnico - Universidade Federal de Santa Maria, e-mail: dinizfronza@yahoo.com.br.

\section{EntomoBrasilis 4 (2): 56-6o (2011)}

Resumo. Com o objetivo de avaliar a eficiência de atrativos alimentares para monitoramento e controle alternativo da mosca do figo [Zaprionus indianus (Gupta)], realizou-se este trabalho. O trabalho foi conduzido no Setor de Fruticultura do Colégio Politécnico da Universidade Federal de Santa Maria, de 22 de janeiro a 22 de abril de 2009 em pomar de figueira (Ficus carica L.). Como modelo de frasco caça mosca utilizaram-se garrafas Pet. de 1L. Como atrativos alimentares, utilizaram-se suco de goiaba, suco de butiá e suco de figo, colocados em diferentes solventes, totalizando para cada frasco, $200 \mathrm{ml}$ de solução. Os valores obtidos foram submetidos à análise estatística. As soluções que apresentaram calda de figo + cachaça $(50 \%+50 \%)$ e calda de figo + água $(50 \%+50 \%)$ apresentaram maiores níveis de captura de indivíduos adultos de $Z$. indianus, sugerindo-se sua utilização no monitoramento da mosca do figo. As soluções a base de álcool são pouco eficientes.

Palavras-Chave: Controle Alternativo; Mosca do figo; Soluções atrativas

\section{Effectiveness of Attractions Food in Capture Zaprionus indianus (Gupta) in Fig Orchard in Santa Maria - RS}

Abstract. Aiming to evaluate the efficiency of food baits for monitoring and alternative control of fig fly [Zaprionus indianus (Gupta)], held this job. The study was conducted in Sector Fruit of the Polytechnic College, University of Santa Maria, from January 22 to April 22, 2009 in fig orchard (Ficus carica L.). As model of trap we used Pet. bottles of $1 \mathrm{~L}$. Guava juice, jelly palm juice and fig juice with different solvents were use as food baits, with 200 $\mathrm{ml}$ solution per bottle. The values were subjected to statistical analysis. The solutions fig juice + rum $(50 \%+50 \%)$ and fig juice + water $(50 \%+50 \%)$ showed higher levels of adult capture of $Z$. indianus, suggesting its use in fly monitoring. The alcohol-based solutions are inefficient.

Keywords: Alternative Control; Attractive solutions; Fig fly

A mosca do figo, Zaprionus indianus (Grupta), é um drosofilídeo de origem africana, característico de regiões tropicais (VILELA et al. 2000). Sua primeira constatação em pomares de figo, no Brasil, em São Paulo, em 1999, época da safra de figos (Vilela et al. 2000; Raga \& Souza Filho 2003). Os danos causados, no ano de constatação, reduziram a produção em 40\% e a exportação em 80\% (STEIN et al. 2003). Durante as safras subseqüentes, os prejuízos continuaram expressivos e sua disseminação por outros estados aumentou (RAGA \& Souza FilHo 2003). No Rio Grande do Sul seu primeiro registro em 2001 (Silva et al. 2005).

A mosca do figo possui uma ampla adaptabilidade, garantida por uma grande quantidade de substratos necessários para o seu desenvolvimento. Por ser uma espécie de clima tropical, obteve no Brasil ótimas condições climáticas para o seu desenvolvimento, espalhando-se de norte a sul com grande facilidade. A rápida infestação dos frutos na época da safra, associada ao cultivo e comercialização, pode estar ligada a uma série de fatores como: coincidência da introdução da praga com o inicio do cultivo; estimulo da desova da mosca pela colheita na fase de maturação; alto potencial vital e ciclo de vida relativamente curto, associado diretamente a altas temperaturas; safra simultânea com outras culturas, propiciando maior fonte de alimento ao indivíduo (Vilela et al. 200o).

A mosca do figo ataca os frutos de figo no início da maturação, inutilizando-os comercialmente. A postura é feita nas brácteas que circundam o ostíolo, em figos intactos e em inicio de amadurecimento, ou seja, fase de colheita para a exportação
(VILELA et al. 2000).

Aspectos biológicos da mosca do figo foram analisados, em aproximadamente 21 dias se obtém os insetos adultos, o que explica a sua rápida evolução a campo logo após a sua constatação como praga, quando não se aplica medidas de supressão da sua população. Podem ocorrer até 12,4 gerações anuais nas condições de Pelotas-RS (Stein et al. 2003; NAva et al. 2007).

A busca por sistemas eficientes de monitoramento é prioridade para o manejo adequado da praga, tornando-se base para ações de prevenção e controle (RAGA \& SouZA FilHo 2003). Com a presença desta espécie, a cultura do figo, tornase fortemente susceptível ao ataque, promovendo-a ao estágio de praga potencial, gerando uma série de riscos a produtividade e rendimento final da cultura. Alternativas de substratos com eficiência foram propostas, porém estas direcionadas para outras regiões do país, utilizando melaço, associado, a figo triturado e suco de laranja (RAGa \& Souza Filho 2003; RAga et al. 2006).

O objetivo do estudo foi avaliar a eficiência de atrativos alimentares, passíveis de serem produzidos em propriedades, a partir de diferentes frutos, para monitoramento e controle alternativo da mosca do figo.

\section{MATERIAL E MÉTODOS}

O experimento foi conduzido na área experimental de Setor de Fruticultura do Colégio Politécnico da Universidade Federal de Santa Maria - RS ( $29^{\circ} 43^{\prime} \mathrm{S}$ e $\left.53^{\circ} 43^{\prime} \mathrm{W}\right)$, com altitude ao redor de $96 \mathrm{~m}$. Segundo a classificação de KÖPPEN, o clima 
da região é do tipo Cfa, com precipitação anual de $1700 \mathrm{~mm}$ e temperatura média anual de $18^{\circ} \mathrm{C}$, a média das máximas do mês mais quente $32^{\circ} \mathrm{C}$ e das mínimas do mês mais frio de $9^{\circ} \mathrm{C}$ (Buriol et al.1979).

O experimento foi conduzido de 22 de janeiro de 2009 a 22 de abril de 2009 em pomar de figueira (Ficus carica L.) cultivar Roxo de Valinhos no quarto ano de produção. Com espaçamento de 2,5 metros entre linhas e 2,0 metros entre plantas na linha. Não houve aplicação de inseticidas durante o período de estudo. Foram utilizados como modelo de frascos caça mosca garrafas Pet $1 \mathrm{~L}$, com dois furos cada de $8 \mathrm{~mm}$ de diâmetro (Figura 1). Como atrativos alimentares, utilizaram-se suco de goiaba, suco de butiá e suco de figo, colocados em diferentes solventes (água, cachaça e álcool), totalizando para cada frasco, $200 \mathrm{~mL}$ de solução atrativa. As caldas feitas em laboratório, a partir de frutos de goiaba, butiá e figo provenientes da região.

Utilizou-se 12 tratamentos, instalados em blocos ao acaso, com quatro repetições para cada tratamento, totalizando 48 parcelas experimentais com uma planta por parcela. Os frascos com as soluções atrativas foram colocados aleatoriamente no pomar, na parte mediana de cada planta a luz indireta do sol. As soluções atrativas foram trocadas a cada 21 dias. As mostras foram retiradas semanalmente e os indivíduos levados para laboratório para triagem, identificação (VILELA et al. 2000) e análise. Os tratamentos foram: calda de figo e álcool $(25 \%+75 \%)$ (T1); calda de figo e álcool (30\%+70\%) (T2); calda de figo e álcool $(50 \%+50 \%)\left(\mathrm{T}_{3}\right)$; álcool e água $(70 \%+30 \%)(\mathrm{T} 4)$; cachaça colonial (100\%) ( 55$)$; cachaça e água $(70 \%+30 \%)(\mathrm{T} 6)$; calda de figo e cachaça $(30 \%+70 \%)(\mathrm{T} 7)$; calda de figo e cachaça $(50 \%+$ $50 \%)(\mathrm{T} 8)$; calda de figo e água (30\% + 70\%) (T9); calda de figo e água $(50 \%+50 \%)($ T10); calda de butiá e água $(50 \%+50 \%)$ (T11); calda de goiaba e água $(50 \%+50 \%)(\mathrm{T} 12)$.

Os valores de insetos obtidos em cada parcela, durante as 12 semanas de captura, foram submetidos à análise de variância (ANOVA) e comparação de médias pelo teste Tukey $(\mathrm{P}<0,05)$. Além da análise geral dos tratamentos, compararamse individualmente cada atrativo alimentar, nas diferentes concentrações, submetidos à análise da variância e ao teste Tukey $(\mathrm{P}<0,05)$

\section{RESULTADOS E DISCUSSÃO}

No período de amostragem em que as soluções atrativas permaneceram no pomar (treze semanas), capturou-se 7.903 indivíduos adultos de $Z$. indianus, numa razão sexual de três fêmeas para cada dois machos. Os resultados médios de captura dos indivíduos por tratamento são demonstrados na figura 2. O tratamento que empregou calda de figo (50\%) + água (50\%) obteve a maior captura, $24 \%$ dos indivíduos, já o tratamento que empregou calda de figo (50\%) + cachaça (50\%) obteve $22 \%$ dos indivíduos capturados, estes, diferiram-se estatisticamente dos demais tratamentos. Os tratamentos que empregaram calda de figo (30\%) + água (70\%) e álcool (70\%) + calda de figo (30\%) ambos obtiveram 11\% (Tabela 1).

Comparando estes dados com os obtidos por RAGA \& Souza Filho (2003) e RaGa et al. (2006) os valores foram inferiores, demonstrando menor eficiência, porém deve-se salientar vários aspectos como: o tipo de frasco caça-mosca (pet $2 \mathrm{~L}$ contra $1 \mathrm{~L}$ ), o número de furos (12 contra 2), o diâmetro dos furos (10 mm contra $8 \mathrm{~mm}$ ), idade do pomar e volume de calda, que contribuíram aparentemente para este sucesso.

O tratamento que apresentou álcool (70\%) + água (30\%), obteve os menores resultados, apenas 1\% dos indivíduos capturados, o que caracteriza o álcool como substância não preferencial para a mosca. Calda de figo (25\%) + álcool (75\%), obteve pouca atratividade, se relacionado com calda de figo + água $(30 \%+70 \%)$, apenas dois porcento. O tratamento que apresentou calda de figo + álcool $(50 \%+50 \%)$, obteve quatro porcento dos indivíduos capturados, esta pouca atratividade pode estar relacionada ao aumento da concentração de suco de figo devido à evaporação do álcool. Com isso, observava-se grande quantidade de formigas doceiras nos frascos e a ação conjunta destes dois fatores pode ter influenciado para a pouca atratividade da calda.

$\mathrm{Na}$ análise estatística individual dos tratamentos a base de álcool e água, T2 diferiu-se estatisticamente de T1, T3, e T4, o que a torna calda preferencial quando utilizado estes ingredientes (Tabela 2).

As soluções atrativas que possuem água como solvente, caracterizam-se por oferecer algumas vantagens em relação aos demais, são mais econômicas e práticas, por possibilitar um maior período de permanência das caldas no pomar, sem necessidade de trocá-las. Estas podendo ser utilizadas por períodos superiores há 21 dias.

Os tratamentos a base de calda de goiaba e de butiá, possuem pouca atratividade se relacionada com aquelas à base de calda de figo, demonstrando uma preferência alimentar por estas caldas, em pomar de figo. Esta pouca atratividade pode estar relacionada a não combinação entre o solvente e o soluto ou mesmo a concentração da calda. $Z$. indianus, por ser uma espécie polífaga, encontra no butiazeiro e goiabeira (Silva et al. 2005; LovaGniNo et al. 2008), substratos para o seu desenvolvimento, indicando a potencialidade destes como atrativos. Tornase necessário, o teste com novas concentrações e novos solventes, para avaliar suas eficiências. Isso pode possibilitar a diversificação de atrativos alimentares e viabilizar o seu controle por um maior período efetivo na pequena propriedade rural visto a disponibilidade de frutos ser maior, para preparar as caldas.

Nas treze semanas de experimento os maiores índices de captura se concentraram da quinta a décima semanas de avaliação, atingindo um pico populacional na sétima semana. Período este correspondente ao pico produtivo do pomar. $\mathrm{Na}$ análise dos frutos colhidos constatou-se pouca quantidade de frutos atacados pela mosca, indicando um possível controle das armadilhas instaladas no pomar (Figura 3).

Diversos fatores contribuíram para o aumento populacional da mosca do figo no pomar, dentre eles a proximidade com outras frutíferas, e como conseqüência, grande quantidade de alimento para a mosca. Ao proceder à retirada dos restos culturais dessas frutíferas, a partir da oitava semana, houve uma redução gradativa na quantidade de adultos capturados pelas armadilhas. Observaram-se no pomar indivíduos adultos de $Z$. indianus associados a frutos defeituosos, com má formação, rachados, ou aqueles atacados por pássaros.

Frutos de figo caídos sobre o chão são ótimas fontes de alimento e atuam como sitio para o desenvolvimento da mosca, a simples permanência deste por algumas horas atua como fonte atrativa para adultos da mosca do figo. Estes frutos caídos quando coletados periodicamente, num intervalo não superior a sete dias, período inferior ao desenvolvimento embrionário e larval da mosca (STEIN et al. 2003), podem atuar como armadilhas naturais, promovendo fonte substrato para adultos ovipositarem e a posterior retirada destes, reduzindo o nascimento de indivíduos da mosca. Prática esta que associada a atrativos alimentares podem atuar eficientemente no controle da mosca do figo.

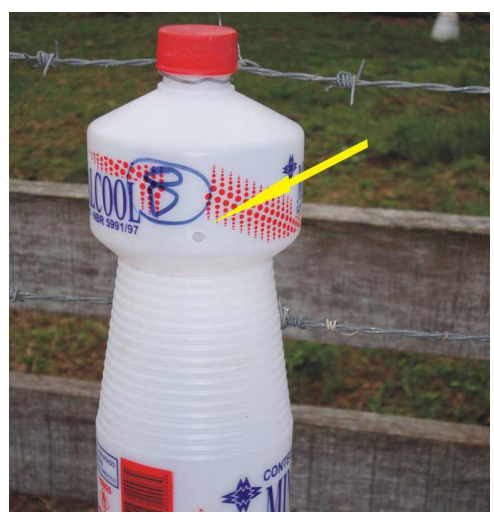

Figura 1. Modelo de frasco caça mosca utilizado em pomar de figo. Seta amarela indica o local da perfuração (furos com $8 \mathrm{~mm}$ de diâmetro). Santa Maria, RS, Safra 2008/o9. 


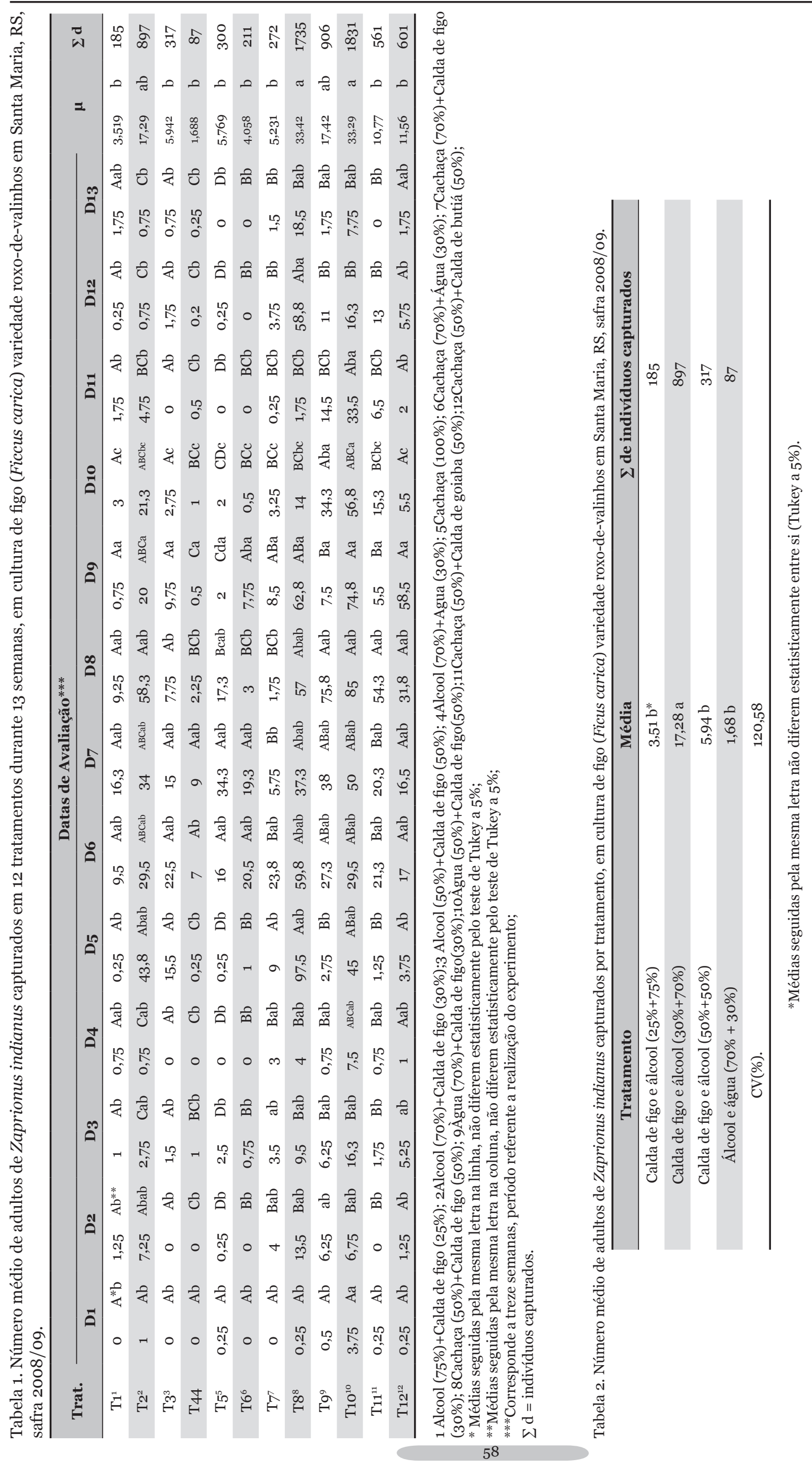




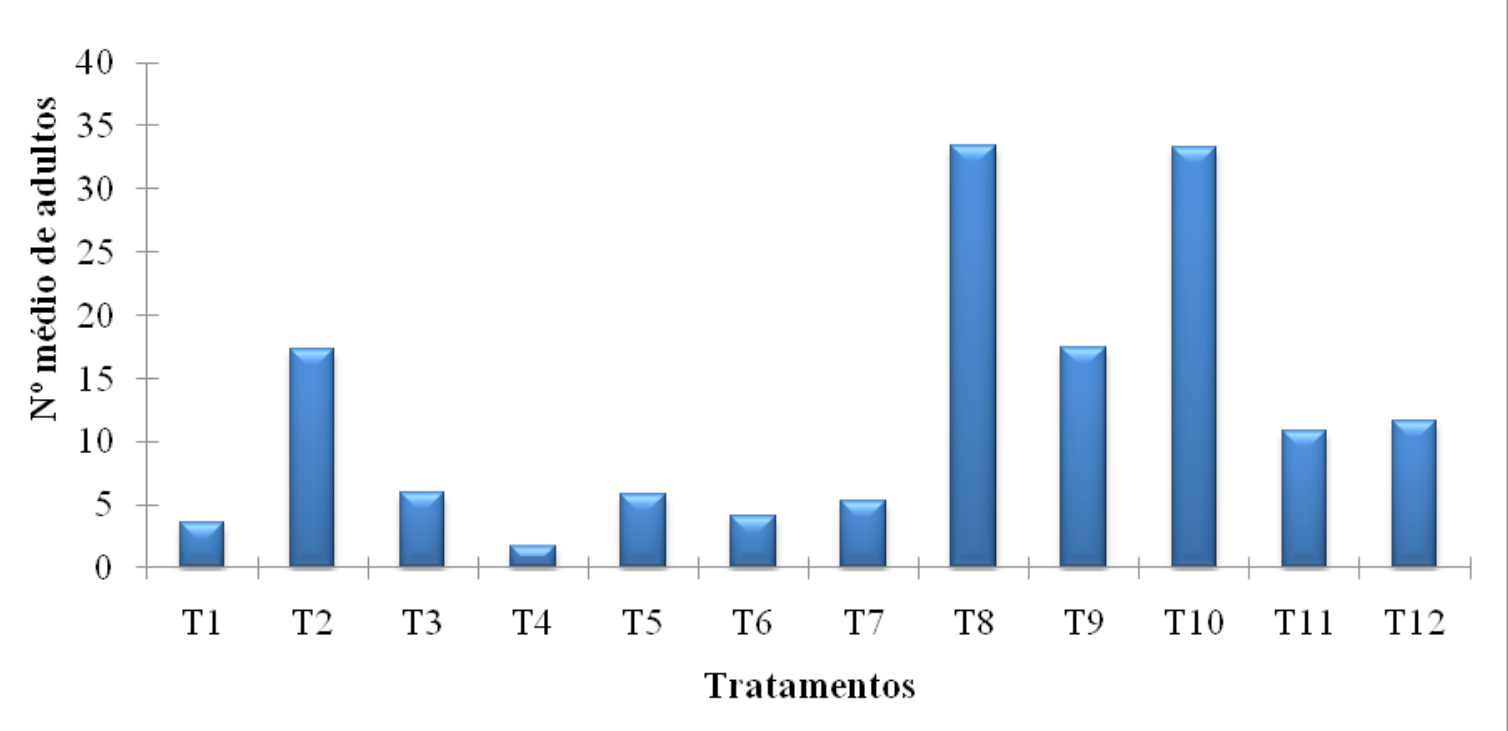

Figura 2. Média de captura da mosca do figo por tratamento (calda de figo e álcool $(25 \%+75 \%)($ T1); calda de figo e álcool $(30 \%+70 \%)($ T2); calda de figo e álcool $(50 \%+50 \%)(\mathrm{T} 3)$; álcool e água $(70 \%+30 \%)(\mathrm{T} 4)$; cachaça colonial (100\%) (T5); cachaça e água $(70 \%+30 \%)(\mathrm{T} 6)$; calda de figo e cachaça (30\% + 70\%) (T7); calda de figo e cachaça $(50 \%+50 \%)(\mathrm{T} 8)$; calda de figo e água $(30 \%+70 \%)(\mathrm{T} 9)$; calda de figo e água $(50 \%+50 \%)(\mathrm{T} 10)$; calda de butiá e água $(50 \%+50 \%)$ (T11); calda de goiaba e água $(50 \%+50 \%)(T 12))$ em pomar de figo, Santa Maria, RS, Safra 2008/09

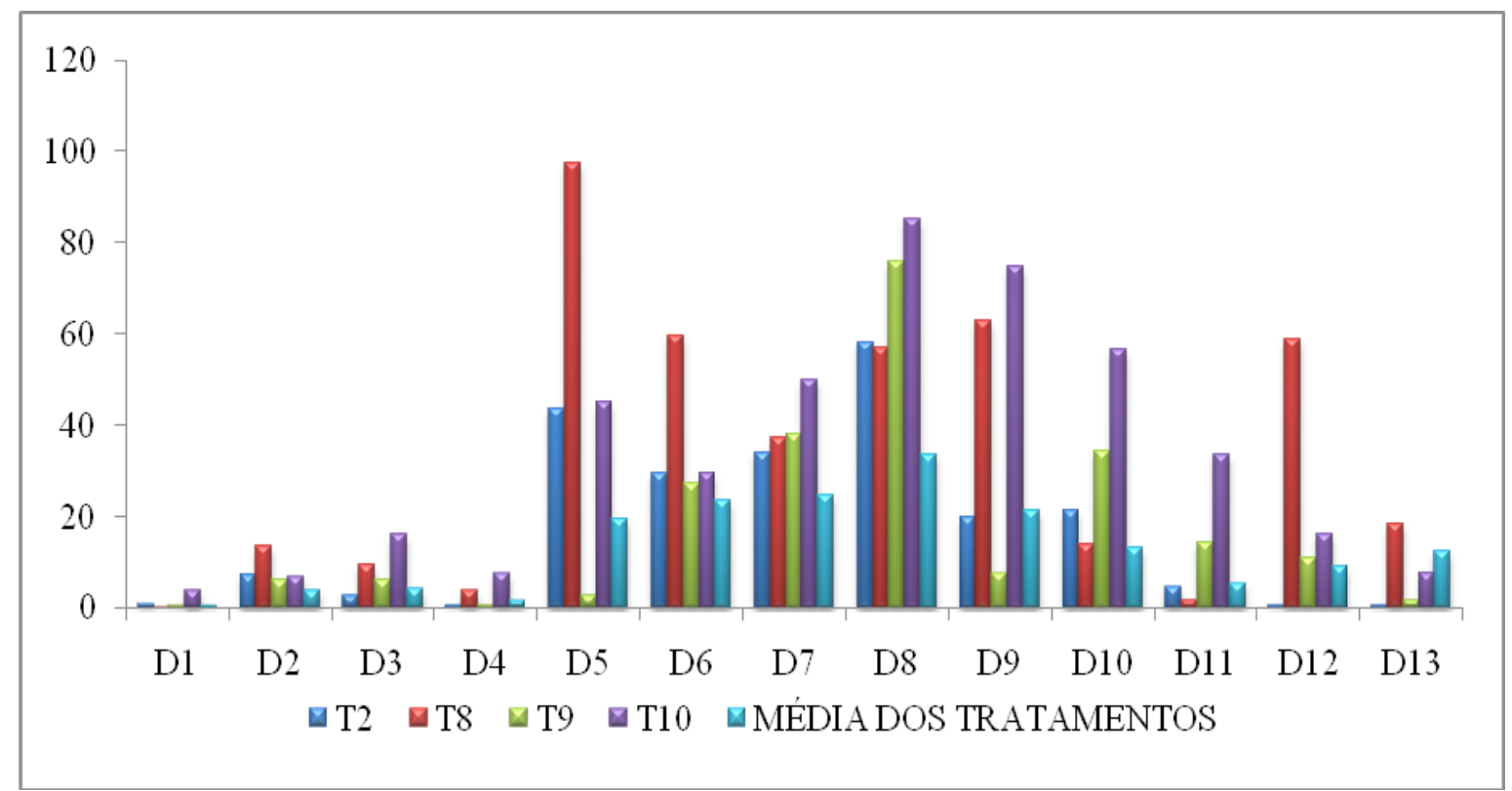

Figura 3. Número médio de adultos capturados, nos quatro tratamentos mais eficientes, durante treze semanas em pomar de figo, Santa Maria, RS. Tratamentos: calda de figo e álcool $(30 \%+70 \%)=$ T2; calda de figo e cachaça $(50 \%+50 \%)=$ T8; calda de figo e água $(30 \%+70 \%)=$ T9; calda de figo e água $(50 \%+50 \%)=$ T10; safra $2008 / 09$.

\section{REFERÊNCIAS}

Buriol, G.A., V. Estefanel, M. Ferreira, A.V. Saccol, F.M. Schneider \& A.B. Heldwein, 1979. Cartas mensais e anuais das temperaturas médias, das médias das temperaturas máximas e das médias das temperaturas mínimas do estado do RS. Revista do Centro de Ciências Rurais, Santa Maria, 9, suplemento, np.

Lavagnino, N.J., V.P. Carreira, J. Menesch, E. Hasson \& J.J. Fanara, 2008. Geographic distribution and hosts of Zaprionus indianus (Diptera: Drosophilidae) in NorthEastern Argentina. Revista da Sociedade de Entomologia da Argentina, 67: 189-192.

Nava, D.E., A.M. Nascimento, C.P. Stein, M.L Haddad, J.M.S. Bento \& J.R.P. Parra, 2007. Biology, thermal requirements, and estimation of the number of generations of Zaprionus indianus (Diptera: Drosophilidae) for the main fig producing regions of Brazil. Florida Entomologist, 90: 495-501.

Raga, A. \& M.F. Souza Filho, 2003. Captura deZaprionus indianus (Gupta) (Dip: Drosophilidae) em frascos de plástico com iscas alimentares na cultura do figo. Revista de Agricultura,
Piracicaba, 78: 323-329.

Raga, A., R.A. Machado, W. Dinardo \& P.C. Strikis, 2006. Eficácia de atrativos alimentares na captura de moscas-das-frutas em pomar de citros. Bragantia, 65: 337-345.

Silva, N.M., C.C. Fantinel, V.L. Valente \& V.H. Valiati, 2005. Ecology of colonizing of the fig fly Zaprionus indianus (Diptera, Drosophilidae) in Porto Alegre, Southern Brazil. Iheringia, Série Zoológica, 95: 233-240.

Stein, C.P., E.P. Teixeira \& J.P.S. Novo, 2003. Aspectos biológicos da mosca do figo, Zaprionus indianus Gupta, 1970 (Díptera: Drosophilidae). Entomotropica, 18: 219-221.

Vilela, C.R., E.P. Teixeira \& C.P. Stein, 2000. Mosca-Africanado-figo, Zaprionus indianus (Diptera: Drosophilidae), p. 48-52. In: Vilela, E.F., Zuchi, R.A. \& F. Cantor (Eds.). Pragas Introduzidas no Brasil. Ribeirão Preto: Holos, 179p.

Recebido em: 11/10/2010

Aceito em: 03/01/2011 


\section{Como citar este artigo:}

Pasini, M.P.B., D. Link \& D. Fronza, 2011. Eficácia de Atrativos

Alimentares na Captura de Zaprionus indianus (Gupta) em

Pomar de Figo em Santa Maria - RS. EntomoBrasilis, 4(2): 56-6o.

www.periodico.ebras.bio.br/ojs

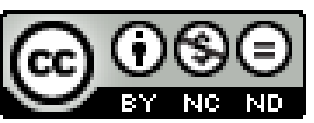

Entono Brasitis

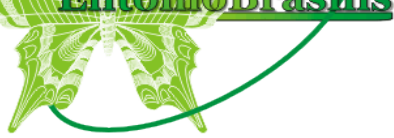

